\title{
Detection of optic nerve damage in ocular hypertension
}

\author{
J E ROSS, A J BRON, B C REEVES, AND P G EMMERSON* \\ From the Visual Science Unit, Department of Ophthalmology, Radcliffe Infirmary, Oxford OX2 $6 A N$
}

SUMMARY Thirty patients with ocular hypertension were tested for contrast sensitivity loss. Seventeen were not on treatment, and thirteen were receiving some form of pressure reducing therapy. The contrast sensitivity results of $63 \%$ of ocular hypertensive eyes were abnormal (greater than 2 SDs from the age matched norm). Thus it appears that contrast sensitivity can detect early visual loss in patients who have normal visual fields and it is suggested that this test might be used as a criterion for therapy in ocular hypertension. There was no significant difference in the intraocular pressures between patients who gave abnormal contrast sensitivity results and those who did not in the untreated group $(p>0 \cdot 05)$, suggesting that intraocular pressure level is a poor predictor of optic nerve fibre damage in patients with ocular hypertension.

An initial phase in chronic simple glaucoma may be identified when there is ocular hypertension (raised intraocular pressure (IOP) greater than $21 \mathrm{mmHg}$ ) but no observable pathological cupping of the optic disc and no glaucomatous field defect. Many ocular hypertensive patients never develop glaucoma, but others are in the early stages of the disorder. ' If the latter patients are not treated, they will develop glaucomatous cupping of the optic disc and visual field defects. The number of patients who develop glaucoma from ocular hypertension has been estimated variously as $0 \cdot 4 \%,{ }^{2} 0 \cdot 5 \%,{ }^{1} 3 \cdot 2 \%,{ }^{3}$ and $17 \cdot 4 \% .^{4}$ When the IOP is persistently above 31 $\mathrm{mmHg}$ the proportion of patients developing glaucoma is thought to be considerably higher, about $40 \%$. $^{5}$ Thus patients with ocular hypertension, to a greater or lesser extent, appear to be more at risk of developing glaucoma than an age matched population with normal pressures.

It has been estimated that field loss may take 10 to 15 years to occur after the onset of raised pressure. ${ }^{6}$ There is therefore a protracted period of time during which optic nerve fibre damage, currently undetectable in vivo, is occurring. Indeed, recent evidence from enucleated eyes has shown that there is optic nerve fibre loss in ocular hypertension in the absence of manifest field loss. ${ }^{7}$ Clearly, more sensitive

Correspondence to Dr J E Ross.

${ }^{*}$ Current address: Visual Sciences Department, Aston University, Gosta Green, Birmingham BE4 7ET methods for differentiating between glaucomatous and non-glaucomatous ocular hypertensive patients are needed to provide specific criteria for diagnosis and therapy.

Many researchers have shown that spatial contrast sensitivity in glaucoma patients can be quite severely depressed $^{*-10}$ and that the reduction in sensitivity can be correlated with other measures of the progression and extent of the disease, such as field loss. ${ }^{11}$ These losses of contrast sensitivity have been found even in patients with early glaucoma who show no central visual field abnormalities - that is, in the central $15^{\circ} .{ }^{10}$ There is also some evidence to suggest that both contrast sensitivity ${ }^{12}$ and colour vision may be impaired in patients with ocular hypertension. ${ }^{12-15}$ Therefore it seemed a logical step to determine whether spatial contrast sensitivity is depressed in some ocular hypertensive patients but not in others. We used an accurate and reliable technique to measure contrast sensitivity ${ }^{16}$ in order to determine its usefulness as an early test of nerve fibre loss in ocular hypertension.

\section{Materials and methods}

SUBJECTS

Patients with ocular hypertension were recruited as they presented to the Eye Clinic. Patients were diagnosed as ocular hypertensive if: (i) IOP was greater than or equal to $22 \mathrm{mmHg}$ on at least two 
occasions in one or both eyes; (ii) there were normal optic discs on ophthalmoscopic examination; (iii) there were goniscopically demonstrated open anterior chamber angles ${ }^{17}$; (iv) there were full Topcon fields to isoptres I2e, I4e, and V4e. Patients were accepted on or off treatment.

Inclusion criteria. Patients were accepted for the study if (i) they fulfilled the above criteria for the definition of ocular hypertension; (ii) their spectacle correction was between -6.00 and +6.00 dioptres inclusive.

Exlusion criteria. Patients were excluded from the study if (i) there was evidence of concurrent eye disease; (ii) there was a personal history of any disorder which might give rise to eye disease in the future, for example diabetes; (iii) there was a diagnosis of glaucoma in the fellow eye.

Thirty patients with ocular hypertension were recruited, 20 males and 10 females. Seventeen patients had not received pressure reducing therapy during the previous three months (group 1) and 13 were receiving treatment (group 2). Details of these patients are summarised in Table 1.

\section{GROUP I}

There were 12 male and five female patients not receiving medication with a mean age of 58.80 (SD 9.73) and 62.00 (SD 4.84) respectively. Eleven of the patients had bilateral ocular hypertension. The majority of the patients presented to Eye Clinic between one month and four years before recruitment, but three patients had been attending for seven, eight, and nine years.

\section{GROUP 2}

There were eight male and five female patients receiving treatment. Their mean ages were 60.75 (SD 8.01) and 63.20 (SD 12.26) respectively. Ten of the patients had bilateral ocular hypertension. Seven patients were on timolol (Timoptol), four were on adrenaline based eye drops, one was on timolol and epinephrine, and one was on pilocarpine. Six of these patients stopped treatment four days prior to the tests. Twelve patients presented to the Eye Clinic between one month and four years before recruitment; the remaining patient had been attending for 10 years.

All patients received a full ophthalmological examination including slit-lamp biomicroscopy, IOP readings recorded with the Goldmann applanation tonometer, and fundus examination. The end point for the measurement of IOP was taken as the mid point of the oscillation of the applanation prism.

\section{VISION TESTS}

All the patients had measurements of visual acuity, Topcon fields, and contrast sensitivity. In addition 11 patients had Octopus field tests.

Corrected distance visual acuity was measured at 6 metres with a Bailey-Lovie test chart. ${ }^{18}$

Visual fields were measured on a Topcon SBP-11H projection perimeter. The background illumination was 32.5 apostilbs and targets $\mathrm{I} 2 \mathrm{e}, \mathrm{I} 4 \mathrm{e}$, and $\mathrm{V} 4 \mathrm{e}$ were used. Octopus fields were also obtained on some patients by means of programme 33 , which assesses the central $30^{\circ}$ of the visual field. The Octopus automatic field tester has a number of advantages when compaired to the Topcon perimeter. These include standardised testing conditions, static randomised presentations of the test targets, and numerical results to which statistical analysis can be readily applied.

For the contrast sensitivity test, static vertical sinewave gratings of variable spatial frequency were generated on a display oscilloscope by a two-channel microprocessor wave form generator, ${ }^{19}$ which was addressed by a DEC PDP 11-23 computer. The contrast of the grating pattern was controlled by a digital to analogue converter on the computer. Contrast is defined as $\left.\left(\mathrm{L}_{\text {max }}-\mathrm{L}_{\text {min }}\right) / \mathrm{L}_{\text {max }}+\mathrm{L}_{\text {min }}\right)$, where $L$ is the luminance of a point on the screen. The procedure for obtaining contrast threshold measurements was based on the method of Wetherill ${ }^{21}$ and has been described in detail elsewhere..$^{916}$

Table 1 Details of patients recruited for the study

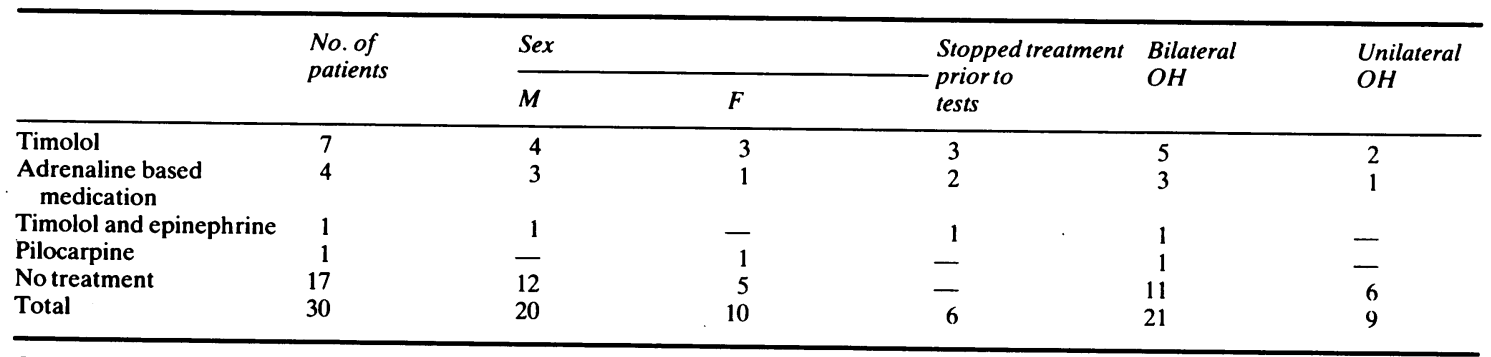

$\mathrm{OH}=$ ocular hypertension. 
Table 2 Intraocular pressure levels in the study groups

\begin{tabular}{|c|c|c|c|c|c|c|}
\hline $\begin{array}{l}\text { Patient } \\
\text { group }\end{array}$ & $\begin{array}{l}\text { No. of } \\
\text { patients }\end{array}$ & $\begin{array}{l}\text { No. } \\
\text { of eyes }\end{array}$ & $\begin{array}{l}\text { Grand mean } \\
I O P\end{array}$ & $S D$ & $\begin{array}{l}\text { IOP at } \\
\text { last visit }\end{array}$ & $S D$ \\
\hline $\begin{array}{l}\text { Group 1 } \\
\text { (notreatment) } \\
\text { Group 2 }\end{array}$ & 17 & 28 & $22 \cdot 80$ & 3.05 & $24 \cdot 50$ & $4 \cdot 34$ \\
\hline (treatment) & 13 & 23 & $22 \cdot 05$ & $2 \cdot 16$ & $21 \cdot 30$ & $2 \cdot 56$ \\
\hline
\end{tabular}

\section{Results}

Monocular visual acuity in both patient groups ranged from $6 / 4$ to $6 / 12$ (mean $6 / 7 \cdot 5$ ). IOP readings are presented in Table 2 . The grand mean pressure is the mean of all recorded pressures since the patient first attended the Eye Hospital. It can be seen from Table 2 that the only IOP measure which differed between the two groups was the group mean IOP at last visit. This result is attributable to the fact that one group was on pressure lowering therapy and the other was not.

A qualitative assessment of the cup to disc ratio was made. In 48 ocular hypertensive eyes this ratio was less than or equal to $\mathbf{0 . 5}$. Two of the remaining eyes had a ratio of 0.8 (same patient), and one eye had a ratio of $0 \cdot 6$, and thus one might have considered these eyes to be abnormal. However, in each case the discs were a good colour and the cups were

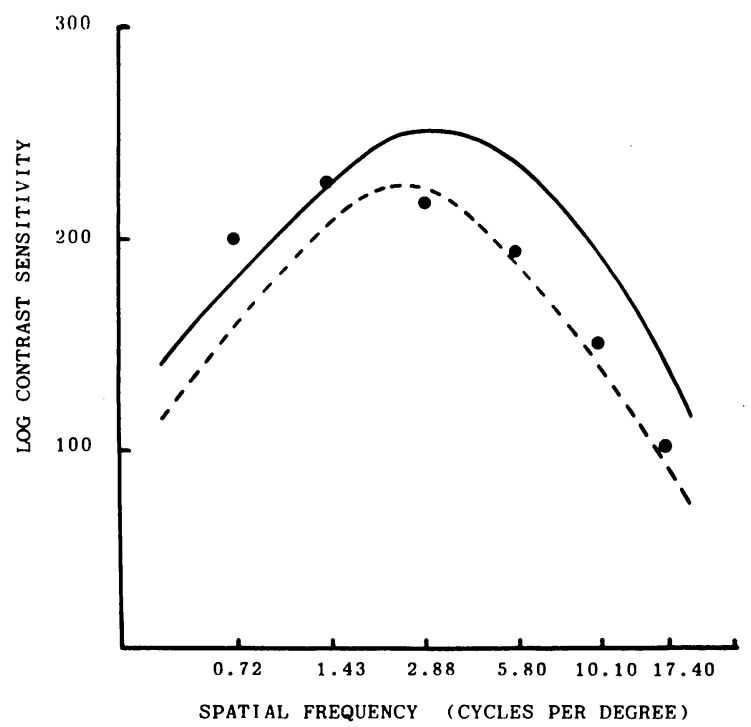

Fig. 1 Contrast sensitivity values (O) for a 72-year-old ocular hypertensive patient with a mild reduction in sensitivity. Note that the area of greatest loss occurs in the middle of the spatial frequency range. $-=$ Mean contrast sensitivity value for age-matched control (71-75 years). ...- = Two standard deviations from the mean. circular, with a healthy rim of tissue at the disc margin. They were therefore considered to be colobomatous discs. Cup to disc ratios exceeded 0.5 in some eyes, which did not fulfil the eligibility criteria for ocular hypertension; details of the findings for these eyes are given in Table $4 \mathrm{~b}$.

The criterion for abnormal contrast sensitivity responses is based on our previous measurements of subjects $(n=70)$, stratified by age, with normal vision. ${ }^{16}$ Our technique of measurement was found to be reliable, with a correlation coefficient of 0.88 or better on a test/retest basis for each spatial frequency. In the present study a response was recorded as abnormal when one or more of the responses to the three spatial frequencies between one and six cycles per degree of visual angle (cpd) were more than two standard deviations (SD) from the age matched mean normal value. By this criterion 20 of the 28 eyes in group 1 and 12 of the 23 eyes in group 2 had abnormal

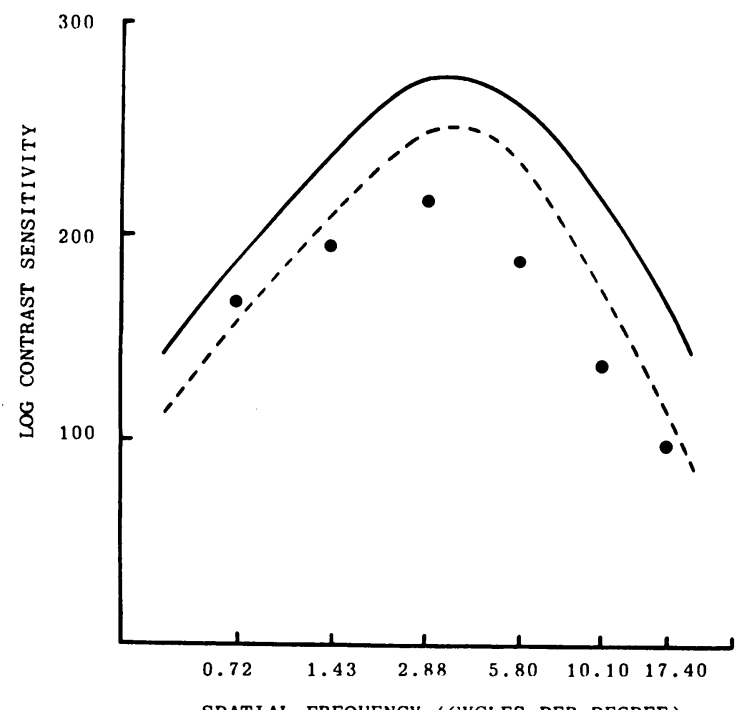

Fig. 2 Contrast sensitivity values (O) for a 51-year-old ocular hypertensive patient with severe loss. The greatest reduction in sensitivity is still found at middle spatial frequencies, but note that a significant loss occurs over a much wider range of spatial frequencies. $-=$ Mean contrast sensitivity value for age-matched control (51-60 years). -..-- = Two standard deviations from the mean. 
Table 3a Contrast sensitivity (CS) results for both eyes of patients who had bilateral ocular hypertension

\begin{tabular}{lll}
\hline $\begin{array}{l}\text { Study } \\
\text { group }\end{array}$ & $\begin{array}{l}\text { No. of } \\
\text { patients }\end{array}$ & CS results \\
\hline 1 & 7 & Both abnormal \\
1 & 3 & Both normal \\
1 & 1 & One abnormal, one normal \\
2 & 4 & Both abnormal \\
2 & 4 & Both normal \\
2 & 2 & One abnormal, one normal \\
\hline
\end{tabular}

contrast sensitivity functions. Examples of the types of contrast sensitivity loss found in the study eyes are presented in Figs. 1 and 2.

Twenty-one patients had bilateral ocular hypertension. Only three of these patients produced asymmetrical contrast sensitivity results (Table 3a), where asymmetry was defined as a normal contrast sensitivity result in one eye and an abnormal result in the fellow eye. The optic discs of these three patients appeared symmetrical and had cup to disc ratios of $0 \cdot 5,0 \cdot 4$, and $0 \cdot 2$. The differences in grand mean IOP between right and left eyes did not exceed $2 \mathrm{mmHg}$.

The remaining nine patients fulfilled the eligibility criteria for entry to the study in one eye only. Of the nine fellow eyes four were normotensive. The other five eyes were excluded for various reasons. Four were ocular hypertensive but showed some narrowing of the superior rim of the optic disc, and one eye was normotensive but had a $2^{\circ}$ nasal step. Such a small defect $\left(<5^{\circ}\right)^{\prime}$ is not classically defined as glaucomatous. These patients were regarded as ocular hypertensive by the referring ophthalmologist on the basis of raised IOP and no visual field loss. However, we recognise the problems involved in including patients in whom the diagnosis of glaucoma is equivocal in one eye and not in the other. For this reason these patients should be distinguished from those who have no supportive evidence of optic nerve damage by disc and visual field change in either eye.

The results for these patients are presented in Table $3 \mathrm{~b}$. All but two of the above nine patients produced asymmetrical results. These two patients were also receiving treatment to both eyes. The first patient was on epinephrine $1 \%$ b.d. and had abnormal contrast sensitivity in the study eye but had symmetrical optic discs. The grand mean IOPs were $21 \mathrm{mmHg}$ in both eyes. In the fellow eye a $2^{\circ}$ nasal step was found on Topcon perimetry, as mentioned above, but normal contrast sensitivity. The second patient was on timolol $0.25 \%$ b.d. and had normal contrast sensitivity in the ocular hypertensive eye, but abnormal sensitivity in the fellow eye. The grand mean IOPs were $22 \mathrm{mmHg}$ in both eyes, and the optic disc in the fellow eye had a cup to disc ratio of $0 \cdot 6$, with narrowing of the superior rim. If the eyes with suspicious discs and possible visual field defects are excluded, there remain four eyes which are normotensive. In each of them contrast sensitivity in the study and fellow eye was similar, with abnormal responses bilaterally in three patients and normal responses bilaterally in one patient. Thus IOP differences between the eyes were not reflected in this result.

Asymmetry between optic discs is usually con-

Table 3b Contrast sensitivity $(C S)$ results for both eyes of patients with unilateral ocular hypertension $(O H)$

\begin{tabular}{llll}
\hline Patient & $\begin{array}{l}\text { Study } \\
\text { group }\end{array}$ & $\begin{array}{l}\text { CS result } \\
\text { for OH eye }\end{array}$ & $\begin{array}{l}\text { CS result } \\
\text { forfelloweye }\end{array}$ \\
\hline 22 & 1 & Abnormal & $\begin{array}{l}\text { Reason for exclusion } \\
\text { offellow eye from study }\end{array}$ \\
28 & 1 & Abnormal & Abnormal \\
26 & 1 & Abnormal & Abnormal \\
27 & 1 & Abnormal & Abnormal \\
30 & 1 & Abnormal & Abnormal \\
29 & 1 & Normal & Normal \\
23 & 2 & Abnormal & Normal \\
25 & 2 & Abnormal & Abnormal \\
24 & 2 & Normal & Abnormal \\
\hline
\end{tabular}

Table 4a Contrast sensitivity (CS) results of bilateral ocular hypertensive patients who had asymmetrical optic discs

\begin{tabular}{rlllll}
\hline Patient & $\begin{array}{l}\text { Study } \\
\text { group }\end{array}$ & Right eye & & Left eye \\
\cline { 3 - 5 } \cline { 4 - 5 } & & C/Dratio & CS result & C/D ratio & CS result \\
\hline 12 & 1 & $0 \cdot 5$ & Normal & $0 \cdot 6$ & Normal \\
21 & 1 & $0 \cdot 4$ & Normal & $0 \cdot 3$ & Normal \\
4 & 2 & $0 \cdot 3$ & Abnormal & $0 \cdot 4$ & Abnormal \\
10 & 2 & $0 \cdot 3$ & Abnormal & $0 \cdot 4$ & Abnormal \\
\hline
\end{tabular}


Table 4b Contrast sensitivity (CS) results of unilateral ocular hypertensive patients who had asymmetrical optic discs

\begin{tabular}{|c|c|c|c|c|c|}
\hline \multirow[t]{2}{*}{ Patient } & \multirow{2}{*}{$\begin{array}{l}\text { Study } \\
\text { group }\end{array}$} & \multicolumn{2}{|l|}{ OHeye } & \multicolumn{2}{|l|}{ Felloweye } \\
\hline & & $C / D$ ratio & CS result & $C / D$ ratio & CS result \\
\hline 26 & 1 & 0.4 & Abnormal & 0.6 & Abnormal \\
\hline 27 & 1 & 0.3 & Abnormal & $0 \cdot 1$ & Abnormal \\
\hline 28 & 1 & 0.3 & Abnormal & 0.6 & Abnormal \\
\hline 22 & 2 & 0.3 & Abnormal & 0.6 & Abnormal \\
\hline 24 & 2 & 0.4 & Normal & 0.6 & Abnormal \\
\hline
\end{tabular}

sidered to be significant in the diagnosis of glaucoma. ${ }^{121}$ Therefore we looked at the contrast sensitivity performance of nine patients who had optic disc cup asymmetry. The results are presented in Tables $4 \mathrm{a}$ and $4 \mathrm{~b}$. Interestingly, all but one patient produced symmetrical contrast sensitivity results. However, this paradoxical result may reflect the small cup to disc ratio differences between the two eyes or the qualitative aspect of the assessment rather than a genuine lack of correlation between optic disc appearance and contrast sensitivity.

An Octopus perimeter enabled us to perform automated field measurements on 13 eyes in 11 patients. However, even when this more rigorous procedure was used, the visual fields in 10 eyes were normal; the other three eyes showed early glaucomatous field defects. These defects were relative arcuate scotomata, in each case adjoining the blind spot. In two of the three eyes Octopus field testing revealed increased thresholds in the foveal area. In these three eyes contrast sensitivity was abnormal (Table 5). The three patients with Octopus field defects were in group 1 (no treatment). In two of the cases the IOPs (both the grand mean and the IOP recorded on the day of the test) were only marginally raised, the highest reading being $23.5 \mathrm{mmHg}$. The third patient was found to have pressures of 36 and $28 \mathrm{mmHg}$ in the right and left eyes respectively and he was placed on timolol drops $0.5 \%$ b.d. to both eyes.

It might be argued that the reason for the high proportion of patients with abnormal contrast sensitivity was poor selection, and that if a more rigorous visual field criterion had been used some patients would have been diagnosed as having chronic simple glaucoma. The above mentioned results from an Octopus automated perimeter on a sample of the eyes included in the study show that this is unlikely to have been the case.

The relationship between contrast sensitivity and the grand mean IOP was examined. In the untreated group there was no significant difference between the mean IOPs of the 20 eyes with reduced sensitivity and the eight which produced normal responses $(t=0 \cdot 199, \mathrm{df}=26, \mathrm{p}>0 \cdot 05)$. In the treatment group 12 eyes produced attenuated contrast sensitivity
Table 5 Contrast sensitivity (CS) and visual field results of patients who had Octopus perimetry

\begin{tabular}{lll}
\hline & Normal CS & Abnormal CS \\
\hline Normal Octopus fields & 1 & 9 \\
Abnormal Octopus fields & 0 & 3 \\
\hline
\end{tabular}

responses, and 11 were within normal limits. Again, differences in grand mean IOPs for the two responses were not statistically significant $(t=1 \cdot 8757, \mathrm{df}=21$, $\mathrm{p}>0.05)$.

\section{Discussion}

In this study $63 \%$ (32 eyes) of the ocular hypertensive eyes showed an impairment of central spatial contrast sensitivity. Individual loss of contrast sensitivity was not related to the grand mean IOPs, and a number of patients with unilateral ocular hypertension who had consistently normotensive fellow eyes nevertheless showed impaired contrast sensitivity in both eyes.

It is accepted that some patients with ocular hypertension will develop chronic simple glaucoma. Those patients with higher pressures (for example, greater than $30 \mathrm{mmHg}$ ) do appear to be at greater risk of developing manifest chronic simple glaucoma, ${ }^{5}$ but when all levels of raised IOP are considered the number of ocular hypertensive patients developing chronic simple glaucoma is surprisingly low. ${ }^{22}$ However, until criteria exist for determining which patients are likely to suffer visual damage, all patients with raised IOP have to be considered at risk.

More than half of our ocular hypertensive patients had defective contrast sensitivity. $71 \%$ of eyes in group 1 and $52 \%$ in group 2 performed at a level which was more than 2 SDs from the age matched norm for one or more spatial frequencies. These abnormal results were obtained from eyes with good visual acuity and full Topcon fields. Surprisingly, of the 11 patients who had Octopus field tests (a more rigorous method of field testing) only three were found to have changes which might be indicative of glaucoma. Both the contrast sensitivity test and the 
Octopus field test sample the central $10-15^{\circ}$ of vision, but it appears that contrast sensitivity is the more sensitive measure of function for central retina.

It is apparent from our results that a far greater proportion of our patients showed reduced contrast sensitivity (and, presumably, optic nerve damage) than might have been expected on the basis of previous reports. ${ }^{812}$ One reason for this discrepancy might be that our control population, used for aged matched comparisons, was not representative. In the present study the comparisons are based on a control population of 70 subjects ( 31 male and 39 female) stratified by age ${ }^{16}$ Moreover, these control data have been supplemented by data from a further 100 normal subjects whose contrast sensitivities fall well within the limits defined by the earlier population. It seems likely, therefore, that in the standardised conditions of the present study a depression of sensitivity greater than 2 SDs below the mean is likely to represent abnormality.

An alternative explanation is that some aspect of the selection of our ocular hypertensive group, either the inclusion/exclusion criteria or the way in which patients were referred, meant that our patients were more at risk of developing optic nerve damage than another group of ocular hypertensive patients not selected in the same manner. This is a possibility, though we have been unable to determine what aspect of the selection/referral procedure could account for our results.

Early glaucomatous defects are typically thought to occur outside the central $5-10^{\circ}$ of the visual field..$^{23}$ However, there is increasing evidence suggesting that vision in central retina is abnormal in ocular hypertension and early glaucoma. Colour vision defects which involve central vision have been reported in ocular hypertension by Lakowski et al. ${ }^{13}$ and in glaucoma and ocular hypertension by Fishman et al. ${ }^{14}$ and Adams et al. ${ }^{15}$ Depression of foveal sensitivity and a generalised depression of the whole central field in patients with ocular hypertension have been reported by Lichter and Standardi ${ }^{24}$ and Anctil and Anderson. ${ }^{25}$ Our own results confirm that depression of central visual thresholds can occur in ocular hypertension, and we suggest that this should be taken as evidence of early optic nerve fibre damage.

The psychophysical data are supported by the in-vitro work of Quigley et al., ${ }^{7}$ who examined the relationship between visual field defects and optic atrophy demonstrated by histology. The histological examination was carried out on enucleated eyes ante or post mortem. Definite focal losses of ganglion cells and optic nerve fibres were found in cases of eyes suspected of having glaucoma even though perimetry had demonstrated no field loss in vivo. The decrease in the total number of nerve fibres below the mean control number varied from $12 \%$ to $40 \%$ in these patients.

In the presence of glaucoma one might expect the eye with the more advanced cupping or visual field defect to have a greater loss of contrast sensitivity. Such an association has been reported by Atkin et al. ${ }^{12}$ and Ross" in glaucoma patients. One might also expect there to be a relationship between IOP and contrast sensitivity loss. In our ocular hypertensive patients no association of this kind was found. All but three patients with bilateral ocular hypertension $(85 \%)$ produced symmetrical contrast sensitivity results (that is, both eyes were either normal or abnormal) despite the fact that in most cases there were pressure differences between the patients' right and left eyes. It is also surprising that seven of the nine patients in whom only one eye satisfied the criteria for ocular hypertension produced symmetrical results, since four of these seven had normotensive fellow eyes and three of these four produced abnormal results. From the point of view of screening, infrequent IOP measurements alone may be a poor indicator of the existence of optic nerve fibre damage. When considering the cause of optic nerve fibre damage in normotensive fellow eyes the inference may be that periods of raised pressure in the fellow eyes have been missed.

From these findings it is clear that contrast sensitivity detects visual loss earlier than rigorous field testing. It is therefore unwise to assume that optic nerve fibres in ocular hypertensive eyes are undamaged if the visual field appears to be full. Our results suggest that the value of ocular pressure measurement for predicting early optic nerve damage is limited, though its use as part of a battery of clinical tests is still important.

Traditionally clinicians do not put a patient on to treatment on the basis of raised IOP alone, unless the pressure is very high (for example, $>30 \mathrm{mmHg}$ ). Usually more emphasis is placed on optic disc appearance and perimetry. In this study we found that there was no obvious relationship between the level of IOP over a wide range and contrast sensitivity loss in either group. This result was hardly surprising in the case of group 2, because, if therapy is effective, the IOP of all of the treated patients should be within normal limits irrespective of the progression of the disease. However, the finding that there is no significant difference in mean IOP between untreated patients who appear to have visual loss and those who do not is very interesting, and shows that IOP measurement, over a wide range, is a very poor indicator of optic nerve fibre damage. It confitms that the policy of not treating patients on the basis of IOP alone is prudent. However, our contrast sensitivity 
results on patients with normal discs and fields show that, if treatment is delayed until manifest signs can be observed (that is, disc changes and/or field defect), extensive visual loss may already have taken place. This is reinforced by recent histopathological studies. $^{\text {? }}$

In this study we investigated a group of patients with raised ocular pressure but with no observable optic disc changes and no field loss. This appears to be a uniform group and, with the exception of patients with very high pressures, the clinician has no specific criterion for treating some patients and not treating others. Our test divides these patients into two groups, those with normal contrast sensitivity and those with abnormal contrast sensitivity. We believe that reduced contrast sensitivity reflects early optic nerve damage and that contrast sensitivity measurements might therefore be used as a criterion for therapy in ocular hypertension.

It has not yet been demonstrated directly that contrast sensitivity is a valid measure of optic nerve damage. However, this could be done simply by a longitudinal study of ocular hypertensive patients. At the same time it would also be interesting to see whether treatment of these patients with pressure reducing therapy is effective in halting and possibly reversing any optic nerve damage that may be occurring, shown by contrast sensitivity loss.

Until such a study has been carried out, we feel it is very important that patients who are referred with raised IOP should undergo rigorous tests of central vision, such as contrast sensitivity and colour vision, in addition to routine visual field testing.

This study was supported by Oxford Regional Health Authority grant number 81/2, RNIB grant DG/POBS/DET/RG, and also by the Visual Research Trust.

\section{References}

1 Armaly MF. Ocular pressure and visual fields. Arch Ophthalmol $1969 ; 81: 25-40$.

2 Graham PA. The definition of pre-glaucoma. A prospective study. Trans Ophthalmol Soc UK 1968; 88: 153-65.

3 Perkins ES. Bedford Glaucoma Survey. II. Rescreening of the normal population. Br J Ophthalmol 1973; 57: 186-92.

4 Walker WM. Ocular hypertension. Follow up of 109 cases from 1963 to 1974. Trans Ophthalmol Soc UK 1974; 94: 525-34.
5 David R, Livingston DG, Luntz MH. Ocular hypertension-a long-term follow up of treated and untreated patients. $\mathrm{Br} J$ Ophthalmol 1977; 61: 668-74.

6 Goldmann. H. Some basic problems of simple glaucoma. Am J Ophthalmol 1959; 48: 213-20.

7 Quigley HA, Addicks EM, Green WR. Optic nerve damage in human glaucoma. Arch Ophthalmol 1982; 100: 135-46.

8 Arden GB, Jacobson J. A simple grating test for contrast sensitivity-glaucoma screening. Invest Ophthalmol Vis Sci 1978; 17: 23-32.

9 Ross JE. The functional effects of visual disorder. Oxford, D Phil thesis, 1983.

10 Ross JE, Bron AJ, Clarke DD. Contrast sensitivity and visual disability in chronic simple glaucoma. Br J Ophthalmol 1984; 68: 821-7.

11 Ross JE. Clinical detection of adnormalities in central vision in chronic simple glaucoma using contrast sensitivity. Int Ophthalmol in press.

12 Atkin A, Wolkstein M, Bodis-Wollner I, Anders M, Kels B, Podos S. Interocular comparison of contrast sensitivities in glaucoma patients and suspects. Br J Ophthalmol 1980; 64: 858-62.

13 Lakowski R, Bryett J, Drance M. A study of colour vision in ocular hypertensives. Can J Ophthalmol 1972; 7: 86-9.

14 Fishman GA, Krill AE, Fishman M. Acquired color defects in patients with open-angle glaucoma and ocular hypertension. Mod Probl Ophthalmol 1974; 13: 335-8.

15 Adams AJ, Rodic R, Husted R, Stamper R. Spectral sensitivity and color discrimination changes in glaucoma and glaucomasuspect patients. Invest Ophthalmol Vis Sci 1982; 23: 516-24.

16 Ross JE, Clarke DD, Bron AJ. The effect of age on contrast sensitivity function: uniocular and binocular findings. $\mathrm{Br} J$ Ophthalmol 1985; 69: 51-6.

17 Gorin G, Posner A. Slit lamp gonioscopy. Baltimore: Williams and Wilkins, 1967: 70-84.

18 Bailey IL, Lovie JE. New design principles for visual acuity letter charts. Am J Optom Physiol Opt 1976; 53: 740-5.

19 Taylor P. A microprocessor controlled gratings generator. J Physiol (Lond) 1978; 284: 20-1P.

20 Wetherill GB. Sequential estimation of quantal response curves. $J R$ Statist Soc, Series B 1963; 25: 1-48.

21 Spaeth GL. Morphological damage of the optic nerve. In: Heilmann K, Richardson KT, eds. Glaucoma. Conceptions of a disease; pathogenesis, diagnosis, therapy. Philadelphia: Saunders, 1978: 138-56.

22 Crick RP, Daubs JG. Epidemiological aids to clinical decision making in primary open-angle glaucoma. Int Ophthalmol 1980; 3: 37-41.

23 Anderson DR, Drance SM, Galin MA, et al. Visual defects in glaucoma: 10. In: Symposium on glaucoma. Trans New Orleans Acad Ophthalmol. St Louis: Mosby, 1975: 190-209.

24 Lichter PR, Standardi CL. Early glaucomatous visual ficld defects and their significance to clinical ophthalmology. Doc Ophthalmol Proc Ser 1979; 19: 111-8.

25 Anctil J-L, Anderson DR. Early foveal involvement and generalized depression of the visual ficld in glaucoma. Arch Ophthalmol 1984; 102: 363-70. 